\title{
Preoperative planning and simulation for pedicle screw insertion using computed tomography-based patient specific volume rendering combined with projection fluoroscopy
}

\begin{abstract}
Background: It is still difficult to perform pedicle screw insertion on patients with abnormal spine morphology. There is an urgent need to provide projection fluoroscopy images to surgeons to practice pedicle screw insertion.

Methods: The simulation software uses a volume rendering algorithm to generate a three-dimensional spine, and it uses a digitally reconstructed radiograph algorithm to simulate anteroposterior and lateral radiographs as the intraoperative image for minimally invasive surgery.

Results: Pedicle screw insertion can be accomplished using a bone drill and multidimensional pedicle screws, and can be exhibited or evaluated in both two and three dimensions. Simultaneously, advanced expert preoperative planning can be shown in system.

Conclusion: A simulator that develops a three-dimensional, patient-specific volume rendered simulation combined with the projection fluoroscopy for pedicle screw insertion is introduced. The simulator can help the surgeon to plan the operation trajectory and can help to predict the complex situation of the operation.
\end{abstract}

Volume 2 Issue I - 2017

\author{
Guoli Song, Jianda Han, Hua Bai,Yiwen Zhao, \\ Xiaozhuang Liu \\ State Key Laboratory of Robotics, Shenyang Institute of \\ Automation, Chinese Academy of Science, China
}

\begin{abstract}
Correspondence: Guoli Song, State Key Laboratory of Robotics, Shenyang Institute of Automation, Chinese Academy of Science, China; Email songgl@sia.cn
\end{abstract}

Received: October 31, 2016 | Published: March 24, 2017

Keywords: pedicle screw, surgical simulator, preoperative planning, expert evaluation

\section{Introduction}

Pedicle screw insertion has been a standard processes for stabilizing the spine ${ }^{1}$ Spinal surgery can be classified as either minimally invasive or open, based on differences in the size of incisions: minimally invasive surgery implants the pedicle screw based on intraoperative fluoroscopic images to ensure the entry point and trajectory, and open surgery implants the pedicle screw based on identification of anatomic landmarks; open surgery needs experienced surgeons. In both minimally invasive and open surgery, the location of the screw can be observed using a postoperative computed tomography (CT) image to evaluate the accuracy of the screw placement to improve the implant method. ${ }^{2-4}$ The procedure of pedicle screw insertion is complicated and has a steep learning curve. Several institutions have reported the complication rates for pedicle screw surgery from 3\% to $55 \%$, with a rate of malpositioning from $1.5 \%$ to $6.7 \% .^{5-13}$ and breach rates from $1.5 \%$ to $58 \% .^{12,14,15,16}$ However, these results were not comprehensive due to a lack of reports from small medical centers and newer surgeons. The breach rates are related with the years of experience of the surgeon; overall breaches and medial breaches are $12.9 \%$ and $8.0 \%$, respectively, for surgeons with less than five years of experience, and they are $10.8 \%$ and $3.5 \%,{ }^{17}$ respectively, for surgeons with more than five years of experience. ${ }^{17}$ These results illustrate that the medial breach rate was significantly lower for the experienced surgeons.

Advanced tools may promote the accuracy and safety needed to help the surgeon perform the surgery. However, there are limitations to these tools, and the surgeon must still rely upon experience with the landmarks of the spine to feel when the screw has appropriately inserted. With the development of image-guide techniques and imaging technology, more high-resolution images could be provided to the surgeon, but these images are almost always two-dimensional image scans that lack anatomical information. Thus, even if the surgeon uses advantageous tools such as an image-guided system, an experienced surgeon is required for preoperative planning, especially when the anatomical structure is complex and abnormal. Once the preoperative planning is complete, the surgeon has to carry out the surgical procedure based on his or her experience, for which hand feel for the tool is an important aspect. Previously, some authors have developed a pedicle screw simulator using noncommercial software platforms. ${ }^{18-21}$ They used a three-dimensional model to simulate the surgical operation; however, it is not suitable with more modern approaches because of the development of image technology. C-arms that capture projection fluoroscopy images are a common image capture device in the operation room, and an O-arm or iso-center $\mathrm{C}$-arm that captures CT scans is used in some hospitals. Accordingly, a simulator should display projection fluoroscopy and CT reslices for the surgeon to more accurately simulate the surgical process.

The objective of the current work was to develop a simulator with the following features

i. Load patient-specific CT scan and generate three-dimensional volume model. 
ii. Generate fluoroscopy images.

iii. Import surgical tools.

iv. Simulate the surgical procedure and show the preoperative planning.

v. Professionally evaluate the surgeon's preoperative plan by comparing to an expert's plan.

vi. Have a force feedback simulator that provides an approximately true force.

These features could help surgeons to perform the insertion of pedicle screws based on patient-specific CT scans and to overcome difficulties that they may encounter during a real surgical procedure. The simulator could be defined as a preoperative planning system or a surgeon training system..$^{22}$ In open surgery, the surgeon could use the three-dimensional model to simulate the procedure by recognizing the anatomic landmark, ensuring the final position of the pedicle screw, and assessing the consequence of the surgery through a simulated axial CT scan image. In minimally invasive surgery, the surgeon could confirm the entry points and trajectory through an intraoperative fluoroscope image; after the implant procedure has concluded, the surgeon can also ensure the final position of the pedicle screw and assess the consequence of the surgery through an axial CT scan image. The surgeon could repeatedly simulate the surgical procedure to gain experience; they can simultaneously implement the individual surgical procedure to improve the accuracy of the surgery.

\section{Materials and methods}

\section{Overview}

The simulator was mainly developed using a volume rendering algorithm of a series of two-dimensional computed tomography (CT) images with the medical standard image format DICOM 3.0. Due to the characteristics of CT images, a threshold-filtering algorithm, which was able to eliminate muscular and adipose tissues, was used to model the anatomy of the bony structures. Thus, a three-dimensional model of a plastic spine model and an individual patient's (female, age 50 , with lumbar disc protrusion between L4 and L5 vertebral body) spine was built separately. Some surgical instruments, including bone drill and pedicle screws which were built by software Solid works have been imported into the system to provide more complete details of the relevant operation. Simultaneously, the simulated frontal and lateral radiograph images and simulated variable-angle and variableposition CT scan images are shown in the system using real-time positioning and posturing of movable instruments consistent with the three-dimensional model.

\section{Software use}

The software allows prediction of where to drill a hole and where to insert pedicle screws. The simulator first loads a patient's specific CT scans and generates a volume rendering spine model to help the surgeons to map the anatomy structure, after which CT reslices and radiograph images are generated that could assist the surgeons with diagnosis. Moreover, the tool will be displayed on the threedimensional CT model, simulated two-dimensional CT scan, and simulated radiograph image. The surgeon is able to operate the handle to control the surgical simulation. Simultaneously, the force feedback which reverses the direction of pedicle screw is displayed as a progress bar while drilling or inserting the pedicle screw. After completing the simulation, preoperative planning for the specific patient is complete. If the simulator is used for training an inexperienced surgeon, an expert operation is required. First, the expert operation finished the surgical process and records the trajectory of pedicle screw. Then, the inexperienced surgeon finished the surgical process independently and the system compares the trajectory between the inexperienced surgeon's and experts'. The distance and angle error of the trajectory will be considered to calculated the final professional evaluation system scores the preoperative planning (Figure 1).

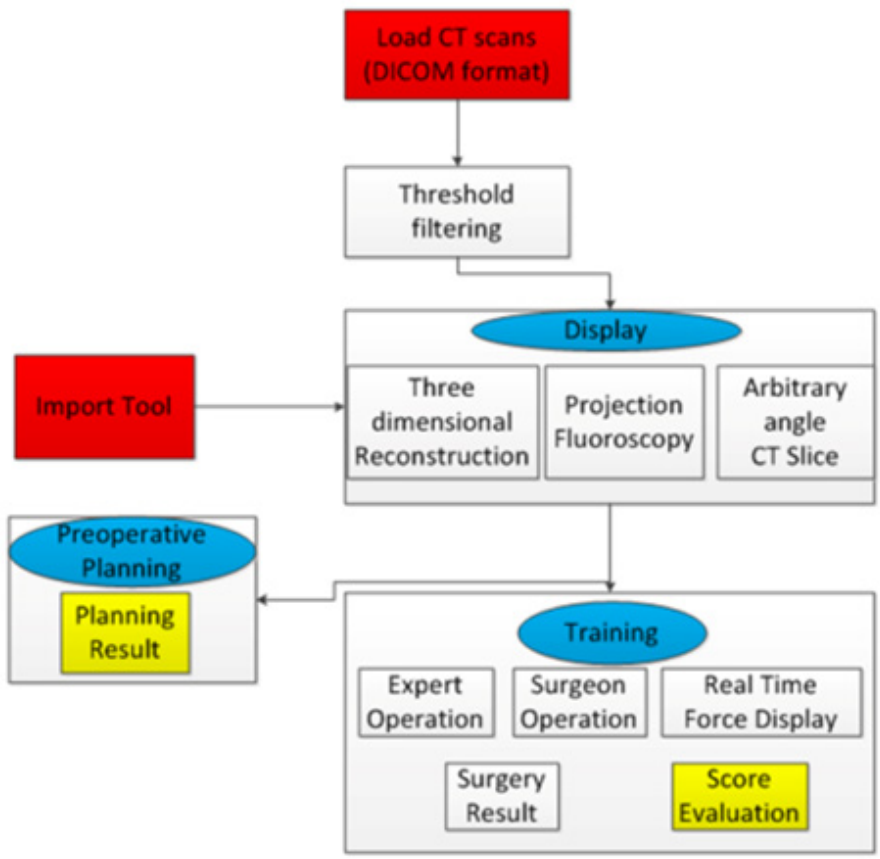

Figure I The framework of the simulator.

\section{Software and hardware requirements}

The simulation hardware consists of a Lenovo D20 graphics workstation and a static joystick. The pedicle screw insertion simulator program is written in $\mathrm{C}++$ language and includes a visualization toolkit (VTK 5.10) and Insight Segmentation and Registration Toolkit (ITK 3.20) library. The platform is set up on Qt (4.8.2), which is a cross-platform application and user interface framework.

\section{Simulation requirements and processes}

A spinal CT scan (DICOM format) with a pixel size of $0.375 \times 0.375$ $\mathrm{mm}$ and a slice thickness of $0.625 \mathrm{~mm}$ is used for the simulation, and all sizes of CT scans are acceptable for the simulator. However, we recommend high-resolution images to obtain more precise anatomical details. After the images are obtained, the threshold filter is used to retain the bony tissue structure in $\mathrm{CT}$ scans. A ray-casting volume rendering algorithm is used to reconstruct the three-dimensional spinal model. At the same time, a projection fluoroscopy image is generated by the Digitally Reconstructed Radiographs (DRRs) algorithm. Another process is the arbitrary angle slice of the threedimensional model to generate $\mathrm{CT}$ images. The three-dimensional spinal models, DRRs, and CT reslices can be displayed individually or simultaneously, the pseudo code of simulator was shown in Table 1. 
Table I The pseudo code of simulator

\section{Load CT data}

Reconstruction three-dimension spine model

Rebuild CT reslice image and digital radiation reconstruction

Load virtual surgical device

Show the device in all the view

Connect the feedback joystick with the virtual surgical device

Move the joystick plan the trajectory of virtual device

judge the grayscale at the top of the virtual surgical device and calculate the force

Feed back the force to joystick

\section{Results}

\section{Three-dimensional reconstruction, fluoroscopy, and $\mathrm{CT}$ reslice}

projection

The simulation software uses a volume rendering algorithm to generate a three-dimensional spine is shown in (Figure 2). Once the CT scans for four vertebral bodies were loaded, the three-dimensional volume rendering spine model, projection fluoroscopy, and CT reslice of the CT image were generated in less than 45 seconds. (Figure 3) Preoperative planning of drilling and inserting pedicle screw with spine model, projection fluoroscopy, and CT reslice. Once the threedimensional volume rendering spine model, projection fluoroscopy, and CT reslice were displayed in the system, surgical tools were imported into the simulator for the future operation. The surgical tools included the bone drill and various sizes of pedicle screws. The process was accomplished by both plastic spine and real patient's spine. The coordinate of virtual illustrated the accuracy of the system (Figure 4). In the open surgery condition, the surgeon was able to use the three-dimensional model as the training environment and drill the pedicle and insert the pedicle screw, after which the surgeon was able to obtain the final position of the pedicle screw in both the CT slice and radiograph image. Conversely, in the minimally invasive surgery condition, the surgeon was able to use two-dimensional frontal and lateral radiograph images as the training environment and drill the pedicle and insert the pedicle screw, after which the surgeon was able to obtain the final position of the pedicle screw in both the CT slice and three-dimensional model.
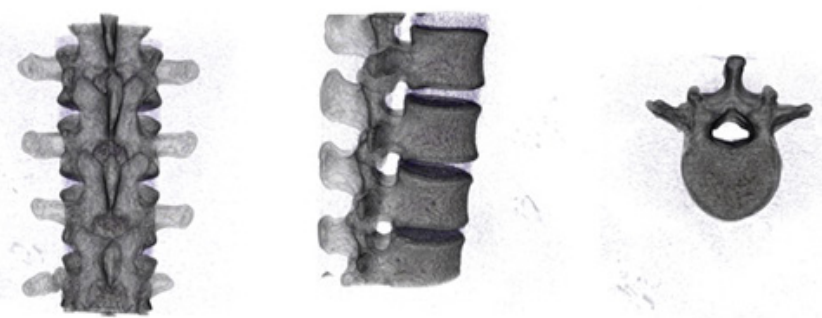

Figure 2 Three-dimensional Model of Spine.

\section{Real-time force feedback}

The different density of bone was reflected in CT scans as a difference in the gray scale. Thus, this gray scale change indicated where different tips of tools were needed in the simulation due to thechanges in bone density. The change was shown by a process bar in the software and was able to be observed by the surgeon (Figure 5).

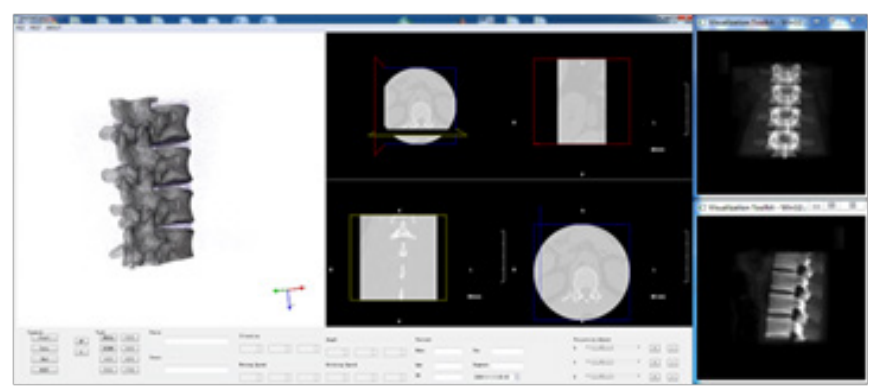

Figure 3 Preoperative planning of drilling and inserting pedicle screw with spine model, projection fluoroscopy, and CT reslice.

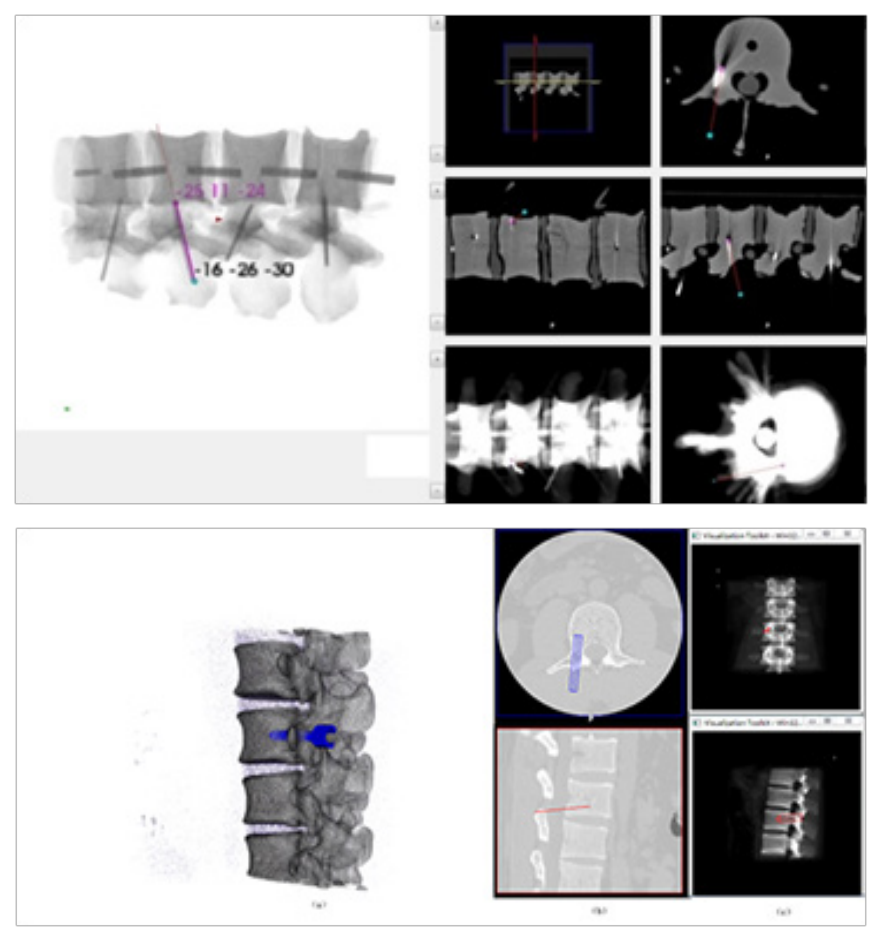

Figure 4 Result of inserting pedicle screw with simulator (a: three-dimensiona spine), (b:CT reslice; $C$ : projection fluoroscopy)

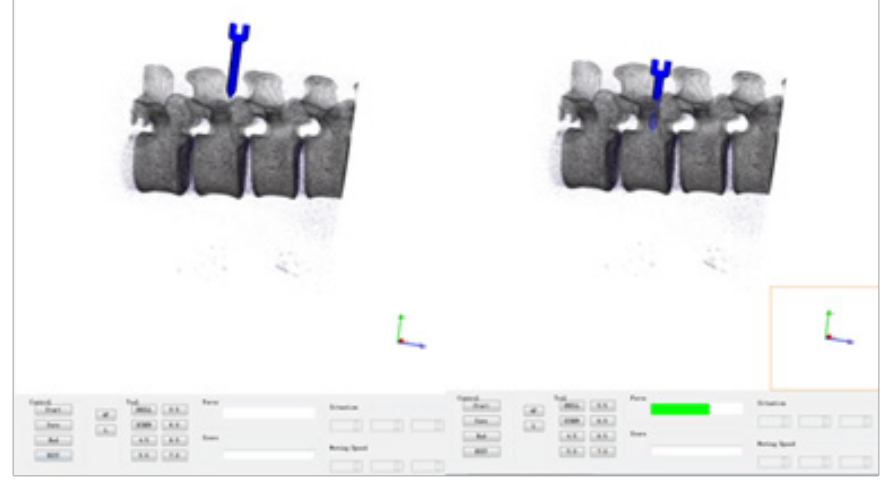

Figure $\mathbf{5}$ The real-time force feedback (left: no feedback; right: the feedback ratio was shown in green bar).

\section{Expert assessment and score}

First, an expert or experienced surgeon planned the trajectory and entry points; then, the software recorded the resulting plan result as the criterion. Next, the surgeon was able to use the simulator to accomplish the surgery procedure. Finally, the surgeon's result and 
expert's result were displayed using the software, and the software was able to conduct the score or evaluation according to the errors between the surgeon's result and the expert's result (Figure 6).
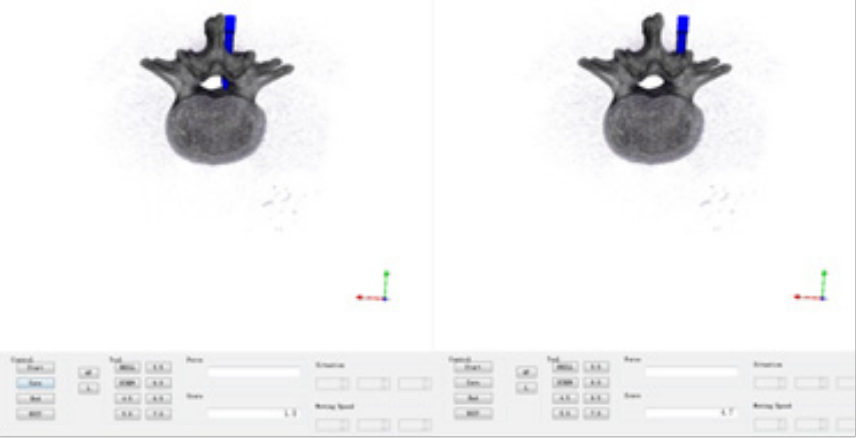

Figure 6 Expert assessment with score (left: malpositioning with lower score I.5; right: a right position similar with expert with high score 4.7).

\section{Discussion}

With the development of surgical technology, spinal fusion surgery with pedicle screw fixation is being used more widely. This reduces the safety by raising the potential risk of surgical procedures and creates a steep learning curve for less experienced surgeons. This could further lead to complications such as infections, epidural hematoma, postoperative fracture, and neuralgia, may require otherwise unnecessary revision surgery, and possibly lead to failure of fusion. ${ }^{21}$ The aim of the simulator and software is to help the surgeon complete the surgery more safely and accurately. A three-dimensional visualization of pedicle screw insertion before surgery may help the surgeon become familiar with the patient's condition, especially in cases of abnormal morphology or other complexities, and thus may reduce the risk of complications. ${ }^{20}$ The three-dimensional view may help the surgeon to ensure the relative position between the surgical tools and a patient's vertebra-the projection fluoroscopy view is consistent with what is customarily used. The CT reslice may also help the surgeon to ensure the position at an arbitrary angle in an arbitrary section. Surgical trainees could learn to repeat the technique repeat at a lower cost and with a curtailed learning curve. The trainee could use the three-dimensional model to diagnose and promote understanding of the anatomy structure, then simulate the surgical procedure, including screw choice, as well as ensure the entry point and pedicle screw insertion point. During pedicle screw insertion, force feedback data would be displayed on the screen in the form of a process bar. Finally, an expert evaluation will be carried out by the software.

When facing complex and abnormal spine morphology such as scoliosis or spinal fractures, pedicle screw insertion is challenging for a surgeon. Complications may lead to catastrophic consequences such as infection, epidural hematoma, postoperative fracture, neuralgia, and even paralysis. Some surgical auxiliary equipment, such as surgical navigation, may reduce complication rates and breach rates, and some surgery navigation systems, such as Spine Assist, can reduce the rate of malpositioning. ${ }^{23-25}$ There is an obvious delineation in the success rate of surgery with and without navigation (4.6\% vs. $13.4 \%$, respectively). ${ }^{23}$ Although the intra operative image could only promote the accuracy over a small range compared with navigation, the high price of the navigation system and complexity of the operation will restrict the system from being used in all hospitals. In the future, the simulator could further combine a hardware system to train and guide the surgeon to implant pedicle screws, thus improving the accuracy of implanting the pedicle screw during actual surgery.

The simulator was tested in an orthopedics department by medical students at Xin-qiao Hospital, Third Military Medical University. The students were divided into two groups with or without the simulator to insert the pedicle screw. The results will be reported in the near future. Currently, the force feedback using the CT gray scale and is shown on the screen, but this is not consistent with the actual surgical environment. Thus, a force feedback joystick, which could simulate force by collision detection, is undergoing development and will be added to the system soon. Additionally, once the registration is added into the system, the preoperative planning will be displayed in an intraoperative image that will guide the surgeon during pedicle screw insertion in the operation room.

\section{Conclusion}

The development of computer technology enables easier medical simulation. The diversity of images requires more diversity in medical simulation. A variety of medical images will allow the surgeon to better understand the anatomy of the patient. The simulator can help the surgeon to plan the operation trajectory and can also help to predict the complex situation of the operation. At present, although the simulator can only be used preoperatively, it provides an obvious benefit to the surgeon, especially one who is less experienced. Further development of the simulator may improve the assistance that surgeons need to safely and accurately complete the operation.

\section{Acknowledgements}

This work was supported in part by National Natural Science Foundation of China (Grant No. 61333019)

\section{Conflict of interest}

The author declares no conflict of interest.

\section{References}

1. Sterba W, Kim DG, Fyhrie DP, et al. Biomechanical analysis of differing pedicle screw insertion angles. Clin Biomech (Bristol, Avon). 2007;22(4):385-391

2. Wang MY, Williams S, Mummaneni PV, et al. Minimally invasive percutaneous iliac screws: initial 24 case experience with CT confirmation. Clin Spine Surg. 2016;29(5):E222-225.

3. Jones-Quaidoo SM, Djurasovic M, Owens RK, et al. Superior articulating facet violation: percutaneous versus open techniques. J Neurosurg Spine. 2013;18(6):593-597.

4. Taghva A, Attenello FJ, Zada G, et al. Minimally invasive posterior atlantoaxial fusion: a cadaveric and clinical feasibility study. World Neurosurg. 2013;80(3-4):414-421.

5. Esses SI, Sachs BL, Dreyzin V. Complications associated with the technique of pedicle screw fixation - a selected survey of abs members. Spine. 1993;18(15):2231-2239.

6. Castro WH, Halm H, Jerosch J, et al. Accuracy of pedicle screw placement in lumbar vertebrae. Spine (Phila Pa 1976). 1996;21(11):1320-1324.

7. Xu R, Ebraheim NA, Ou Y, Yeasting RA. Anatomic considerations of pedicle screw placement in the thoracic spine Roy-Camille technique versus open-lamina technique. Spine (Phila Pa 1976). 1998;23(9):10651068.

8. Amiot LP, Lang K, Putzier M, et al. Comparative results between conventional and computer-assisted pedicle screw installation in the thoracic, lumbar, and sacral spine. Spine (Phila Pa 1976). 2000;25(5):606-614 
9. Boachie-Adjei O, Girardi FP, Bansal M, et al. Safety and efficacy of pedicle screw placement for adult spinal deformity with a pedicle-probing conventional anatomic technique. J Spinal Disord. 2000;13(6):496-500.

10. Laine T, Lund T, Ylikoski M, et al. Accuracy of pedicle screw insertion with and without computer assistance: a randomised controlled clinical study in 100 consecutive patients. Eur Spine J. 2000;9(3):235-240.

11. Belmont PJ, Klemme WR, Dhawan A, et al. In vivo accuracy of thoracic pedicle screws. Spine (Phila Pa 1976). 2001;26(21):2340-2346.

12. Belmont PJ, Klemme WR, Robinson M, et al. Accuracy of thoracic pedicle screws in patients with and without coronal plane spinal deformities. Spine (Phila Pa 1976). 2002;27(14):1558-1566.

13. Carbone JJ, Tortolani PJ, Quartararo LG. Fluoroscopically assisted pedicle screw fixation for thoracic and thoracolumbar injuries - Technique and short-term complications. Spine (Phila Pa 1976). 2003;28(1):91-97.

14. Suk SI, Kim WJ, Lee SM, et al. Thoracic pedicle screw fixation in spinal deformities - Are they really safe. Spine (Phila Pa 1976). 2001;26(18):2049-2057.

15. Kim YJ, Lenke LG, Bridwell KH, et al. Free hand pedicle screw placement in the thoracic spine: Is it safe. Spine (Phila Pa 1976) 2004;29(3):333-342

16. Rajasekaran S, Vidyadhara S, Ramesh P, et al. Randomized clinical study to compare the accuracy of navigated and non-navigated thoracic pedicle screws in deformity correction surgeries. Spine (Phila Pa 1976). 2007;32(2):E56-64.

17. Samdani AF, Ranade A, Sciubba DM, et al. Accuracy of free-hand placement of thoracic pedicle screws in adolescent idiopathic scoliosis: how much of a difference does surgeon experience make? Eur Spine J. 2010;19(1):91-95.
18. Phillips NI, John NW. Web-based surgical simulation for ventricular catheterization. Neurosurgery. 2000;46(4):933-936.

19. Eftekhar B, Ghodsi M, Ketabchi E, Surgical simulation software for insertion of pedicle screws. Neurosurgery. 2002;50(1):222-223.

20. Yamazaki M, Okawa A, Akazawa T, et al. Usefulness of 3-dimensional full-scale modeling for preoperative simulation of surgery in a patient with old unilateral cervical fracture-dislocation. Spine (Phila Pa 1976). 2007;32(18):E532-536.

21. Klein S, Whyne CM, Rush R, et al. CT-based Patient-specific simulation software for pedicle screw insertion. J Spinal Disord Tech. 2009;22(7):502-506.

22. Xiang L, Zhou Y, Wang H, et al. Significance of Preoperative Planning Simulator for Junior Surgeons' Training of Pedicle Screw Insertion. J Spinal Disord Tech. 2015;28(1):E25-29.

23. Ringel F, Villard J, Ryang YM, et al. Navigation, robotics, and intraoperative imaging in spinal surgery. Adv Tech Stand Neurosurg. 2014;41:3-22.

24. Onen MR, Naderi S. Robotic systems in spine surgery. Turk Neurosurg. 2014;24(3):305-311.

25. Marcus HJ, Cundy TP, Nandi D, et al. Robot-assisted and fluoroscopyguided pedicle screw placement: a systematic review. Eur Spine J. 2014;23(2):291-297. 